\title{
Evidence for composite fermions from the magnetothermopower of 2D holes
}

\author{
P.A. Crump a , B. Tieke b ${ }^{\text {, R.J. Barraclough }}{ }^{\text {a }}$, B.L. Gallagher ${ }^{\text {a,* }}$, R. Fletcher ${ }^{\text {c }}$, \\ J.C. Maan ${ }^{b}$, T.M. Fromhold ${ }^{a}$, M. Henini ${ }^{\text {a }}$ \\ - Department of Physics, University of Nottingham, Nottingham NG7 2RD, UK \\ 'High Field Magnet Laboratory, Nijmegen Univetsity, Nijmegen, The Netherlands \\ - Physics Department, Queen's University, Kingston, Ontario, Canada
}

Received 27 June 1995; accepted for publication 10 August 1995

\begin{abstract}
Abetract
Magnetothermopower measurements of high-mobility 2D hole gases are shown to provide strong evidence for a composite fermion Fermi surface at Landau level filling factor $v=1 / 2$. Novel behaviour at fractional filling factors $1 / 3$ and $2 / 3$ are also observed with thermopower minima evolving into maxima with increasing temperature. This is interpreted in terms of the strong screening of the high-mass composite fermions.
\end{abstract}

Keywords: Electrical transport measurements; Gallium arsenide; Heterojunctions; Magnetic measurements; Quantum effects; Semiconductor-semiconductor heterostructures

\section{Introduction}

When a temperature gradient is applied to a conductor, thermoelectric voltages are generated. The measured longitudinal $\left(S_{x x}=\partial V_{x} / \partial T_{x}\right)$ and transverse $\left(S_{x y}=\partial V_{y} / \partial T_{x}\right)$ thermopowers can give detailed information about the strength of the carrier-phonon coupling as a function of magnetic field. In this study, we use measurements of $S_{x x}$ to study the interaction of acoustic phonons with the composite fermions (CFs).formed in a 2D hole gas:

At a Landau Level filling factor of $v=1 / 2$, the properties of $2 \mathrm{D}$ electrons or holes may' be described in terms of composite Fermions (CFs),

\footnotetext{
* Corresponding author. Fax: +44115 9515180, e-mail: ppzblg@ppnl.nott.ac.uk.
}

consisting of one carrier and two flux quanta. The CFs are predicted to form a new Fermi surface at $v=1 / 2$ where they effectively experience zero field, with the region between $v=1 / 3$ and $v=1$ acting as the integer quantum Hall regime for the new CFs. Using low-field transport analysis, the CFs are found to have enhanced mass and reduced lifetime relative to the zero-field values [1].

The thermopower in hole gases has recently been studied at both high temperatures $(T>1 \mathrm{~K})$ [3] and at very low temperatures $(T<100 \mathrm{mK})$ [4]. In this paper we present thermopower measurements on high-mobility $\mathrm{GaAs}-(\mathrm{Ga}, \mathrm{Al})$ As heterojunction 2D hole gases in the intermediate temperature range $260 \mathrm{mK}-1.2 \mathrm{~K}$. As we will show, this is the appropriate region to study the $\mathrm{CF}$-phonon interaction. The samples used are referred to as samples A and B, grown on wafers 
NU1155 and NU1071, respectively. The samples exhibited persistent photoconductivity after infrared illumination, allowing control of the number density $p_{\mathbf{r}}$.

\section{Results for $B=0 \mathrm{~T}$ and at $v=1 / 2$}

The measured thermopower is generally due to two effects: (i) the diffusion thermopower $S_{\mathrm{d}}$, and (ii) the phonon-drag thermopower $S_{\mathrm{g}} \cdot S_{\mathrm{d}}$ arises from the energy dependence of the conductivity of the carriers while $S_{\mathrm{g}}$ is due to scattering between the non-equilibrium phonons and the carriers [2]. When discussing the temperature dependence of the thermopower of degenerate carriers it is useful to consider the approximate expression [2]

$S=\alpha\left(p_{\mathrm{o}}\right) T+\kappa(T) f\left(T, p_{\mathrm{s}}\right)$,

where $\alpha\left(p_{z}\right)$ is a constant giving the strength of the diffusion term, $\kappa(T)$ is the thermal conductivity, and $f\left(T, p_{s}\right)$ is the term giving the strength of the carrier-phonon interaction.

The influence of the two terms can be seen in Fig. 1a, which shows $S_{x x}$ in sample A measured using DC techniques at zero field and at two number densities. $S / \kappa$ is plotted as a function of temperature to reveal $f\left(T, p_{s}\right)$. In the low temperature limit we expect $\kappa \propto T^{3}$, as is observed (see Fig. 1a, inset). The diffusion term therefore gives $S_{d} / \kappa \propto T^{-2}$, a region clearly seen in Fig. la at temperatures below $300 \mathrm{mK}$. This is the region studied by Bayot et al. [4]. Above $400 \mathrm{mK}$ the phonon drag term dominates, with $S / \kappa \approx f\left(T, p_{0}\right)$. A maximum is seen in $S / \kappa$ at $T \approx 1 \mathrm{~K}$ when $p_{3}=$ $0.93 \times 10^{11} \mathrm{~cm}^{-2}$. This corresponds to resonant phonon absorption across the Fermi circle, i.e. it is a form of Kohn anomaly. The maximum will occur when the dominant phonon wavevector $q_{D}=$ $2 k_{\mathrm{p}}$, corresponding to a phonon back-scattering a carrier. $q_{\mathrm{D}} \propto T$, so increasing number density will move the maximum to larger temperatures, as $k_{\mathrm{F}} \propto \sqrt{p_{\mathrm{v}}}$. Increasing $p_{\mathrm{s}}$ to $1.33 \times 10^{11} \mathrm{~cm}^{-2}$ moves the maximum out of the measurable range. Such features are well known at zero field and are a direct consequence of the existence of a Fermi circle $[2,7]$.

Fig. $1 \mathrm{~b}$ shows the results at $v=1 / 2$ for the same two number densities, again presented as $S / \kappa$. If a true Fermi surface forms, then the functional form should be equivalent to the zero-field case. This is observed, with both the low-field diffusion limit and the resonant absorption maximum being present. The location of the maximum roughly scales with total number density as $\sqrt{p_{a}}$ as expected, although the shift is close to the resolvable limit. A Kohn anomaly in $S / \kappa$ can only occur when a Fermi surface exists. This data therefore gives direct evidence for a Fermi surface at $v=1 / 2$.

The expected positions of the Kohn anomaly maxima estimated from the condition $q_{\mathrm{D}}=2 k_{\mathrm{F}}$ are marked in Figs. 1a and 1b. For a given phonon velocity, one would expect the peaks at $v=1 / 2$ to be higher in temperature by a factor of $\sim \sqrt{2}$ than those at $B=0 \mathrm{~T}$. However, the relative importance of the coupling for transverse and longitudinal phonons (the velocities of which differ by $\sim 2$ ) may have a major influence. Previous studies on $n$-type GaAs-(Ga,Al)As heterostructures at zero field have found the transverse phonons to dominate [2]. However, recent work on hot carrier phonon emission in p-type $\mathrm{GaAs}-(\mathrm{Ga}, \mathrm{Al})$ As heterostructures has found strong coupling between holes and both longitudinal and transverse acoustic phonons at zero field [6]. Our results are consistent with this work, with the measured zero-field Kohn anomaly lying between the positions predicted for interaction with only transverse or only longitudinal phonons. This indicates that holes couple strongly to both types of acoustic phonon. However, as can be seen in Fig. 1b, the position at $v=1 / 2$ agrees well with that expected if the CFs couple predominantly to the transverse phonons. The existence of these Kohn anomaly peaks at $v=$ $1 / 2$ gives direct evidence for a CF Fermi surface. However, it is not immediately obvious why the CFs should couple much more strongly with transverse than with longitudinal acoustic phonons. These results indicate the need for theoretical work on the $\mathrm{CF}$-phonon interaction.

\section{Magnetothermopower}

Thermopower was measured using an $\mathrm{AC}$ pulsed heating technique as a function of field up to $20 \mathrm{~T}$ 


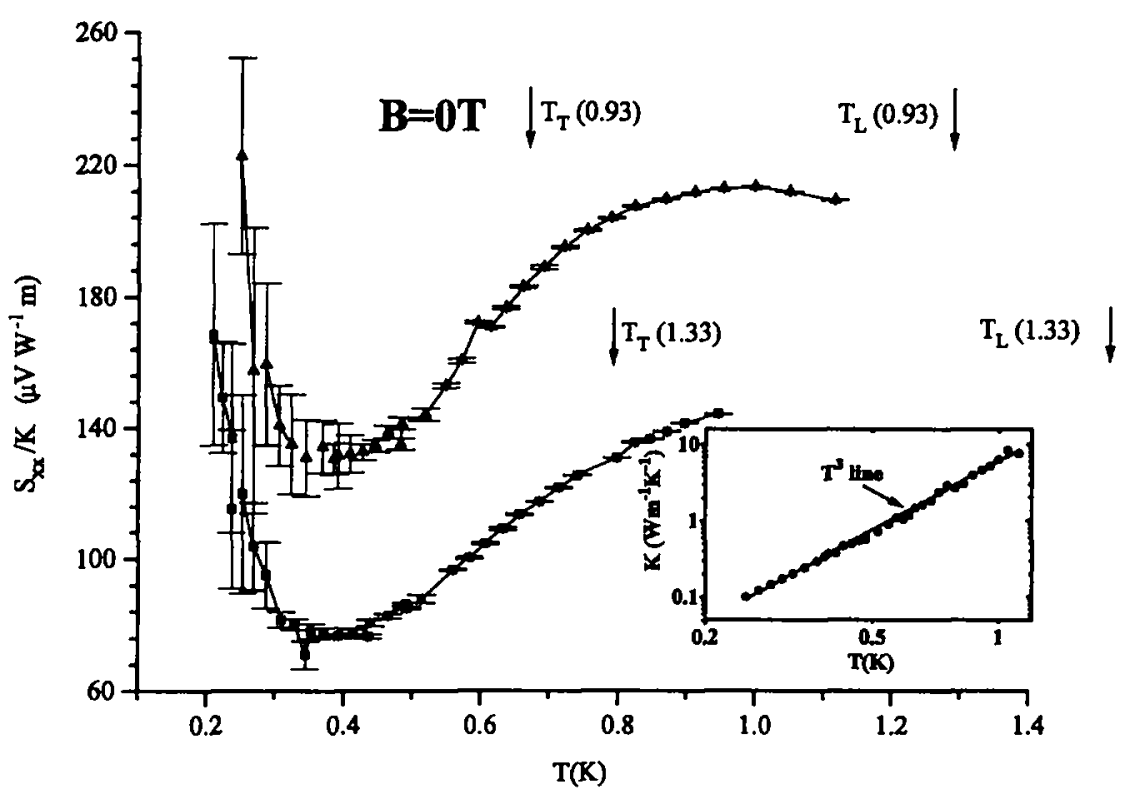

(a)

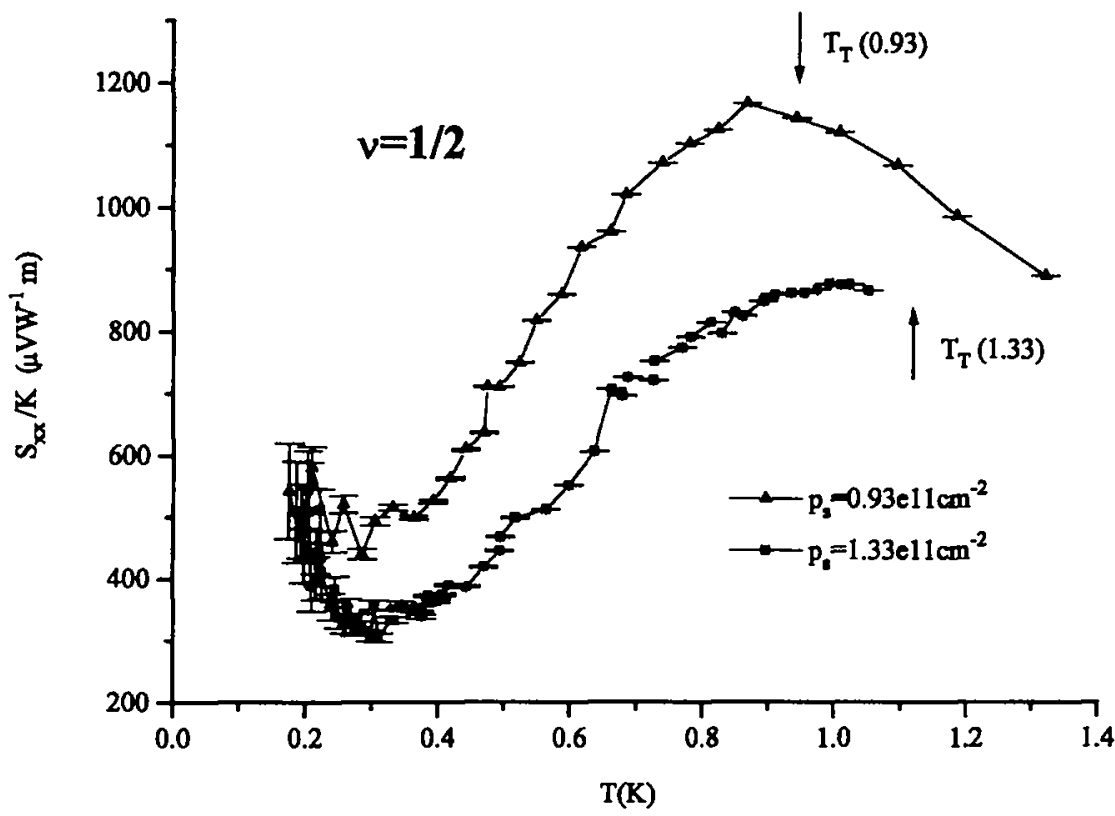

(b)

Fig. 1. (a) Zero-field thermopower of sample A scaled by the measured thermal conductivity at $p_{\mathrm{a}}=0.93 \times 10^{11} \mathrm{~cm}-2(\Delta)$ and at $p_{a}=$

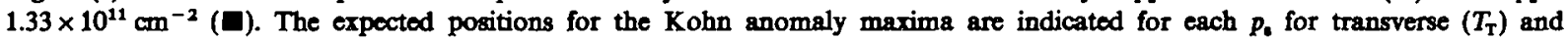
longitudinal $\left(T_{1}\right)$ acoustic phonons. The measured maxima can be seen to lie between the two expected positions. Insert: measured thermal conductivity of sample A. The expected $T^{3}$ dependence is seen, showing the accuracy of the thermometry. (b) Thermopower of sample $A$ at $v=1 / 2$ scalod by thermal conductivity at same number densities as for $B=0 T$. The expected Kohn anomaly maxima position for the transverse acoustic phonons at each $p_{a}$ is indicated. The expected position of the longitudinal phonon maxima is not shown as it lies outside the range of the graph: $T_{L}(0.93)=1.82 \mathrm{~K}$ and $T_{L}(1.33)=2.16 \mathrm{~K}$. 


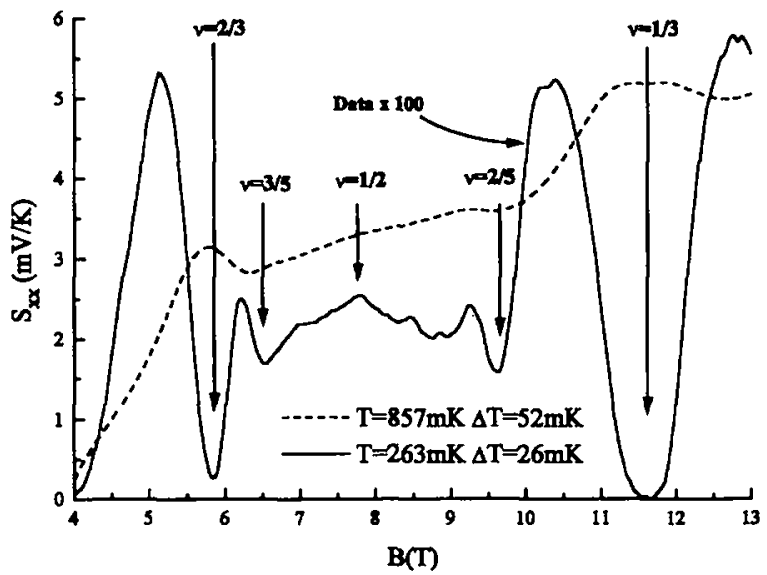

Fig. 2. Magnetothermopower of sample $A$ around $v=1 / 2$ at $T=263$ and $857 \mathrm{mK}$. The absolute magnitude has strongly increased with temperature, so the $263 \mathrm{mK}$ data is increased 100 times so that the high-field and low-field structure can be compared. The evolution from minimum to maximum at $v=$ $1 / 3$ and $v=2 / 3$ with temperature is clear. Note also the striking symmetry in field of the data around $\nu=1 / 2$ at lower $T$.

in a Bitter magnet. Typical results for $S_{x x}$ for sample $A$ are shown in Fig. 2 for $T=857 \mathrm{mK}$ and $236 \mathrm{mK}$. As can be seen, at the highest temperatures $S_{x x}$ maxima form at the $v=1 / 3$ and $v=2 / 3$ fractions. This is an effect unique to holes, fractional $S_{x x}$ minima always being seen in electrons. The fractional size of the $S_{x x}$ features at $v=1 / 3$ and $2 / 3$ is plotted in Fig. 3 as a function of temperature for sample $B$. If the magnitude of this feature is determined by resonant phonon absorption across the energy gap $E_{\mathrm{g}}$, the temperature dependence of the feature size should scale with both $E_{z}$ and the energy at which the phonon number density is a maximum, $1.6 k_{\mathrm{B}} T$. As is seen in Fig. 3, the data at $v=1 / 3$ and $2 / 3$ does indeed show such a scaling.

However, although resonant phonon absorption explains the temperature scaling of the data, it does not explain the functional form of the temperature dependence and should apply equally to electrons where no $S_{x x}$ maximum is seen. In the low temperature limit, the phonon drag term at the fractional states tends to zero since the probability of a phonon exciting a carrier across the energy gap tends to zero. $S_{x x}$ zeroes form and the defined fractional feature size tends to -1 . At the

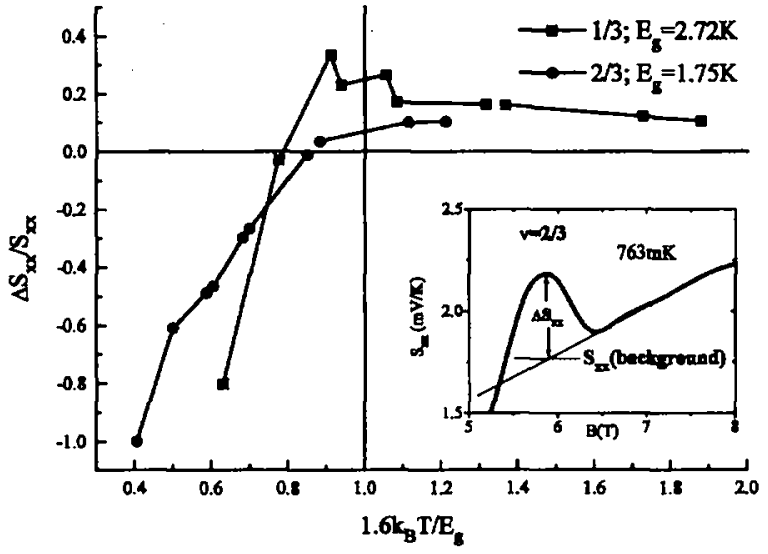

Fig. 3. Evolution of fractional size of feature soen in sample B as a function of scaled temperature. The definition of fractional size is shown in the inset: fractional size $=\Delta S_{x x} / S_{x x}$ (background). This is well defined until the lowest temperatures, where extra resolved structure makes inferring $S_{x x}$ (background) more difficult.

high temperature limit $T \gtrsim E_{\mathrm{g}} / k_{\mathrm{B}}$, the fractional state is unobservable in transport measurements, so the feature size tends to zero.

The striking observation is that in the intermediate temperature regime fractional maxima are observed. The unique feature of the CF holes is their large effective mass, larger than the electron CF mass. Previous theoretical work by Fromhold et al. [2,5] on "normal" fermions showed that the relative phase of the resistivity and $S_{x x}$ depend critically on the strength of the screening. In the phonon drag model, screening of the deformation potential controls the magnitude of the thermopower. Strong screening implies weak carrierphonon coupling and small thermopower. Conversely, weak screening implies a large thermopower. If the density of states at the Fermi energy $D\left(E_{F}\right)$ is large enough, the screening at the center of a Landau level becomes so strong that a minimum forms in the thermopower, with maxima occurring between the Landau levels. The resistivity follows the density of states, so anti-phase $S_{x x}$ and $\rho_{x x}$ arise. Strong Landau level mixing in "normal" holes leads to an enhanced CF hole effective mass, producing an enhanced $D\left(E_{\mathrm{P}}\right)$ for the CFs, which in principle can lead to an antiphase $S_{x x}$. Preliminary calculations of the CF 
magnetothermopower using a reasonable CF mass are able to reproduce $S_{x x}$ maxima at fractional filling, but the results are very sensitive to the exact values chosen for both the mass and the $C F$ scattering time.

\section{Conclusion}

Our results give strong support for the existence of a composite Fermi surface at $v=1 / 2$ and indicate very strong screening of the $\mathrm{CF}$-phonon interaction.

\section{References}

[1] H.C. Manoharan, M. Shayegan and S.J. Klepper, Phys. Rev. Lett. 73 (1994) 3270.
[2] B.L. Gallagher and P.N. Butcher, in: Handbook on Semiconductors, Vol. 1, Eds. T.S. Moss and P.T. Landsberg (Elsevier, Amsterdam, 1992) p. 721.

[3] R.J. Barraclough, T.M. Fromhold, P.J. Rodgers, B.L. Gallagher, P.A. Crump, A. Jezierski and M. Henini, in: Proc. 22nd Int. Conf. on Physics of Semiconductors, Vol. 1, Ed. DJ. Lockwood (World Scientific, Singapore, 1994) p. 819.

[4] V. Bayot, X. Ying M.B. Santos and M. Shayegan, Europhys. Lett. 25 (1994) 613.

[5] T.M. Fromhold, P.N. Butcher, G. Qin, B.G. Mulimani, J.P. Oxley and B.L. Gallagher, Phys. Rev. B 48 (1993) 5326 , and references therein.

[6] R.E. George, K.R. Strickland and AJ. Kent, in: Proc. 22nd Int. Conf. on Physics of Semiconductors, Vol. 1, Ed. DJ. Lockwood (World Scientific, Singapore, 1994) p. 899.

[7] B.L. Gallagher, J.P. Oxley, T. Galloway, M.J. Smith and P.N. Butcher, J. Phys. Condens. Matter 2 (1990) 755. 